OPEN ACCESS

Edited by:

Elisabet Rodby-Bousquet, Lund University, Sweden

Reviewed by:

Reidun Birgitta Jahnsen,

University of Oslo, Norway

Jill Edith Cadwgan,

Evelina London Children's Hospital, United Kingdom

*Correspondence: Cherry Kilbride cherry.kilbride@brunel.ac.uk

tThese authors have contributed equally to this work and share first authorship

Specialty section:

This article was submitted to Pediatric Neurology, a section of the journal Frontiers in Neurology

Received: 26 January 2021 Accepted: 14 April 2021 Published: 12 May 2021

Citation:

Ryan JM, Lavelle G, Theis N, Kilbride $C$ and Noorkoiv M (2021) Patterns of Health Service Use Among Young People With Cerebral Palsy in England. Front. Neurol. 12:659031. doi: 10.3389/fneur.2021.659031

\section{Patterns of Health Service Use Among Young People With Cerebral Palsy in England}

\author{
Jennifer M. Ryan ${ }^{1,2+}$, Grace Lavelle ${ }^{3 t}$, Nicola Theis ${ }^{4}$, Cherry Kilbride ${ }^{2 *}$ and \\ Marika Noorkoiv ${ }^{2}$
}

1 Department of Public Health and Epidemiology, Royal College of Surgeons in Ireland (RCSI) University of Medicine and Health Sciences, Dublin, Ireland, ${ }^{2}$ College of Health, Medicine and Life Sciences, Brunel University London, London, United Kingdom, ${ }^{3}$ Institute of Psychiatry, Psychology and Neuroscience, King's College London, London, United Kingdom, ${ }^{4}$ School of Sport and Exercise, University of Gloucestershire, Gloucester, United Kingdom

Background: Although the provision of healthcare for people with cerebral palsy (CP) is typically focussed on childhood, many people with CP require access to services periodically throughout their life. Few studies have examined patterns of health service use among young people with CP in England. Understanding patterns of use may inform future service development.

Objective: To describe patterns of visits to rehabilitation and medical professionals among ambulatory young people with CP living in England, and identify factors associated with service use.

Methods: Sixty-two young people with CP aged 10-19 years [mean (SD) age 13.7 (2.5) years] in Gross Motor Function Classification System (GMFCS) levels I-III reported visits to a range of health professionals, hospital admissions and visits to the emergency department over a median duration of 34 weeks (min-max: 12-34 weeks). Negative binomial models were used to examine factors associated with number of visits.

Results: Physiotherapists were the most commonly used professional, with $67.7 \%$ of participants visiting a physiotherapist at least once, followed by dentists (66.1\%), general practitioners (48.4\%), occupational therapists (40.3\%) and orthopaedic surgeons (40.3\%). Physiotherapists were also the most frequently visited professional with a total of 473 visits (13.3 visits per person-year). Speech and language therapists (5.0 visits per person-year), occupational therapists (4.5 visits per person-year) and nurses (4.3 per person-year) were the next most frequently visited professionals. Age, GMFCS level, and speech impairment were associated with rate of visits to a physiotherapist.

Conclusions: The proportion of young people who visited medical and rehabilitation professionals during the study period varied considerably depending on the profession. Generally, the proportion of young people using services was low. In the context of limited resources, data on service use in combination with data on unmet need, may support the reorganisation of services to maximise benefits to young people with $\mathrm{CP}$.

Keywords: cerebral palsy, health services, adolescent, rehabilitation, neurological disorders 


\section{BACKGROUND}

Cerebral palsy $(\mathrm{CP})$ is a lifelong condition, with the majority of people with $\mathrm{CP}$ surviving to at least 60 years (1). Although impaired motor function is the core feature, people with $\mathrm{CP}$ can experience a range of associated impairments, such as epilepsy and intellectual disability (2). The type and severity of impairment experienced varies significantly between individuals. People with CP are intensive users of healthcare. Between 2000 and 2014, children and young people with CP in Northern Ireland accounted for $1.6 \%$ of total hospital admissions and $1.6 \%$ of total outpatient appointments, despite representing just $0.3 \%$ of the population (3). Although there is evidence that service use declines from childhood to adolescence and through to adulthood $(4,5)$, this may be because of difficulties accessing services rather than lack of need (6). Adults with CP have an increased risk of chronic physical health conditions, mental health conditions, and falls compared to adults without CP (710). Further, between 18 and $63 \%$ of young people with CP aged 14-18 years report needs in areas such as epilepsy, bone or joint problems, and control of movement, with between 10 and $45 \%$ reporting that their needs are not met (11).

Understanding patterns of health service use and predictors of service use is essential for planning service delivery. However, despite a significant proportion of young people with $\mathrm{CP}$ in the UK reporting healthcare needs (11), there is limited information available regarding service use in this group. A recent report found that, between 2004 and 2014, the rate of GP consultations was $\sim 200$ per 100 person-years among people with CP aged 10-14 years and 250 per 100 person-years among those aged 15-19 years in England (12). Rate of outpatient appointments were approximately 450 per 100 person-years among people aged 10-14 years and 350 per 100 person-years among those aged 15-24 years (12). Rate of inpatient visits were $\sim 50$ per 100 person-years among people aged 10-19 years (12). Another recent study of children and young people with $\mathrm{CP}$ aged 0-24 years in Northern Ireland reported that $68.4 \%$ had at least one hospital admission between 2004 and 2014 and 32.6\% had at least one outpatient appointment between 2010 and 2014 (3). While providing important information using population based datasets, these studies do not describe the range of services used by young people with $\mathrm{CP}$.

A survey of young people in Northern Ireland in 2008 identified the percentage of $12-18$ year olds who visited a range of professionals during a 6-month period, which ranged from $3 \%$ for a psychologist to $92 \%$ for a physiotherapist (5). This study however was limited to non-ambulatory individuals with $\mathrm{CP}$ [i.e., those in Gross Motor Function Classification System (GMFCS) levels IV and V]. As people with CP in GMFCS levels IV and V typically have greater needs and are more frequent users of health services than people in levels I-III $(4,13-15)$, findings may not be applicable to ambulatory people with $\mathrm{CP}$ who constitute $\sim 75 \%$ of the CP population (16).

While these studies provide essential information about service use among young people with $\mathrm{CP}$, there remains gaps in our knowledge about the range of professionals used by young people with CP in the UK. In this paper, we aim to add to current knowledge by describing patterns of visits to rehabilitation and medical professionals among ambulatory young people with $\mathrm{CP}$ living in England, and identifying factors associated with service use.

\section{METHODS}

\section{Sample}

Young people with $\mathrm{CP}$ who participated in a randomised controlled trial examining the effects of a 10 -week progressive resistance training programme were included in this study (17). Participants were recruited from eight National Health Service (NHS) trusts in England, a special education needs school, a University, a primary care organisation in London, national organisations for people with disabilities, and by word of mouth. Young people aged 10-19 years with spastic CP and the ability to walk independently with or without a mobility aid [i.e., Gross Motor Function Classification System (GMFCS) levels I-III] were included in the study. The GMFCS is a five-level classification system (18). Those in GMFCS level I are able to walk and run and climb stairs without assistance. Those in level II are able to walk in most settings but may use a hand-held mobility device indoors or wheeled mobility to travel long distances. Those in level III can walk using a hand-held mobility device but use a wheelchair or powered mobility outdoors. Young people were excluded if they had orthopaedic surgery of the lower limbs in the past 12 months, botulinum toxin type A injections or serial casting in the past 6 months, or insufficient cognition to comply with assessment procedures and the training programme. Approval was obtained from Brunel University London's College of Health, Medicine and Life Sciences Research Ethics Committee and the Surrey Borders Research Ethics Committee (ref: 15/LO/0843). Participants 16 years and over provided written consent. Participants under 16 years provided assent, and parents of participants under 16 years provided written consent.

Assessments were conducted at baseline, 10 and 22 weeks. Demographic and CP-related information was collected at baseline using a standardised questionnaire. Anatomical distribution was described as unilateral or bilateral. Functional mobility was classified according to the GMFCS. Participants selected a statement that best described their mobility based on descriptors of each GMFCS level. Two physiotherapists retrospectively cross-referenced subjective ratings of GMFCS level against video recordings of participants, obtained as part of the baseline assessment. Participants were also asked if they had autism spectrum disorder (ASD), attention deficit hyperactivity disorder (ADHD), behavioural problems, speech problems, epilepsy, or learning difficulties. We combined those with ASD, ADHD or behavioural problems into one category as less than five people reported having ASD and ADHD, respectively.

\section{Outcomes}

The primary outcome in this study was the binary outcome of whether or not an individual used a health service. Secondary outcomes were number of visits, service provider and setting. At each assessment, participants completed a modified Client 
Service Receipt Inventory (CSRI) to assess health service use (19). At the baseline assessment, participants were asked to state the number of times they visited a range of professionals, number of visits to the emergency department, and number of hospital admissions in the past 12 weeks. They were also asked to state if the service was provided by the NHS, school or privately, and they were asked to state the setting they saw the professional in (i.e., clinic, home or school). At the 10 and 22 week assessment, participants were asked to provide the same information considering the time-period since their last assessment (i.e., the previous 10 and 12 weeks). Assistance was provided by the researcher to read the questions if required. Further, the young person was allowed to ask their parent/guardian or researcher for assistance to answer the questions if required.

\section{Analysis}

The distribution of data was examined using histograms, Q$\mathrm{Q}$ plots, and cross-tabulations. Participant characteristics were described as mean and standard deviation (SD), minimum, maximum, frequencies and percentages, as appropriate. We reported the number and percentage of people with at least one visit to each professional, with at least one visit to the emergency department, and at least one hospital admission. Some participants did not complete the CSRI at 10 or 22 weeks. Therefore, to account for the fact that participants were observed for varying lengths of time, we calculated the incidence rate of at least one visit as the number with at least one visit divided by total person-weeks under observation. We also calculated the rate of visits as total number of visits to each professional divided by total person-weeks. For each service, we examined associations between participant characteristics (i.e., age, sex, GMFCS level, distribution, behavioural problems, speech problems, epilepsy, learning difficulties and type of school) and having at least one visit using separate Cox proportional hazards models. For each service, we also examined associations between participant characteristics and number of visits using separate negative binomial models including an offset for person-time. Where there was evidence that the independent variable was associated with the outcome at the level of $\alpha=10 \%$ (i.e., $p<0.10$ ) in unadjusted analyses, we included these variables together in an adjusted model. Where type of school was not associated with the outcome, we additionally included it in adjusted models as we identified that it confounded the association between a number of participant characteristics and service use. We combined visits to a psychologist and psychiatrist when examining associations because only three people reported visiting a psychiatrist. We did not examine associations with visits to a social worker, chiropodist or audiologist because five or fewer young people reported visiting these professionals. Similarly, we did not examine associations with hospital admissions because less than five children were admitted to hospital. Analyses were performed using Stata version 13.

\section{RESULTS}

Sixty-four participants were recruited to the study. Two participants did not complete the CSRI at any assessment and
TABLE 1 | Participant characteristics.

\begin{tabular}{lcc}
\hline & $\boldsymbol{n}(\%)$ & $\begin{array}{c}\text { Mean (SD); } \\
\text { min, max }\end{array}$ \\
\hline Age, yr & 62 & $\begin{array}{c}13.7(2.5) \text {; } \\
10,19\end{array}$ \\
Female & $26(41.9)$ & \\
Male & $36(58.1)$ & \\
GMFCS level & & \\
I & $28(45.2)$ & \\
II & $25(40.3)$ \\
III & $9(14.5)$ \\
Distribution & & \\
Unilateral & $30(48.4)$ \\
Bilateral & $32(51.6)$ \\
Type of school & & \\
Mainstream education & $50(80.6)$ \\
SEN & $12(19.4)$ \\
Presence of additional impairment & \\
Epilepsy & $7(11.3)$ \\
Speech impairment & $14(22.6)$ \\
Learning difficulties & $20(32.3)$ \\
ASD/ADHD/Behaviour impairment & $9(14.5)$ \\
\hline
\end{tabular}

$A D H D$, attention deficit hyperactivity disorder; ASD, autism spectrum disorder; GMFCS, Gross motor function classification system; SD, Standard deviation; SEN, Special education needs.

were therefore excluded from analysis. Sixty-two participants provided data at baseline, 51 participants provided data at 10 weeks, and 50 participants provided data at 22 weeks. Seventyfour percent of participants provided data at all assessments. Participants were observed for a total of 1,854 person-weeks, with a median of 34 weeks per person (min-max: 12-34 weeks). Characteristics of included participants are presented in Table $\mathbf{1 .}$ Participants were aged 10-19 years. The majority were male, with bilateral $\mathrm{CP}$, and in mainstream education.

\section{Patterns of Service Use}

Participants' use of each service is described in Table 2. Physiotherapists were the most commonly visited professional with $67.7 \%$ of participants visiting a physiotherapist at least once, followed by dentists (66.1\%), general practitioners (GPs) (48.4\%), occupational therapists $(40.3 \%)$ and orthopaedic surgeons (40.3\%). Physiotherapists were also the most frequently visited professional with a total of 473 visits (13.3 visits per personyear). Speech and language therapists (5.0 visits per person-year), occupational therapists (4.5 visits per person-year), and nurses (4.3 visits per person-year) were the next most frequently visited professionals. Nine participants (14.5\%) attended the emergency department at least once. The rate of emergency department visits was 0.31 per person-year. Only three participants $(4.8 \%)$ had a hospital admission, resulting in an admission rate of 0.08 per person-year. For all services except for counselling and social work, the majority of participants accessed the service through the NHS (Table 2). The majority of participants 
TABLE 2 | Description of visits to each professional, emergency department visits, and hospital admissions.

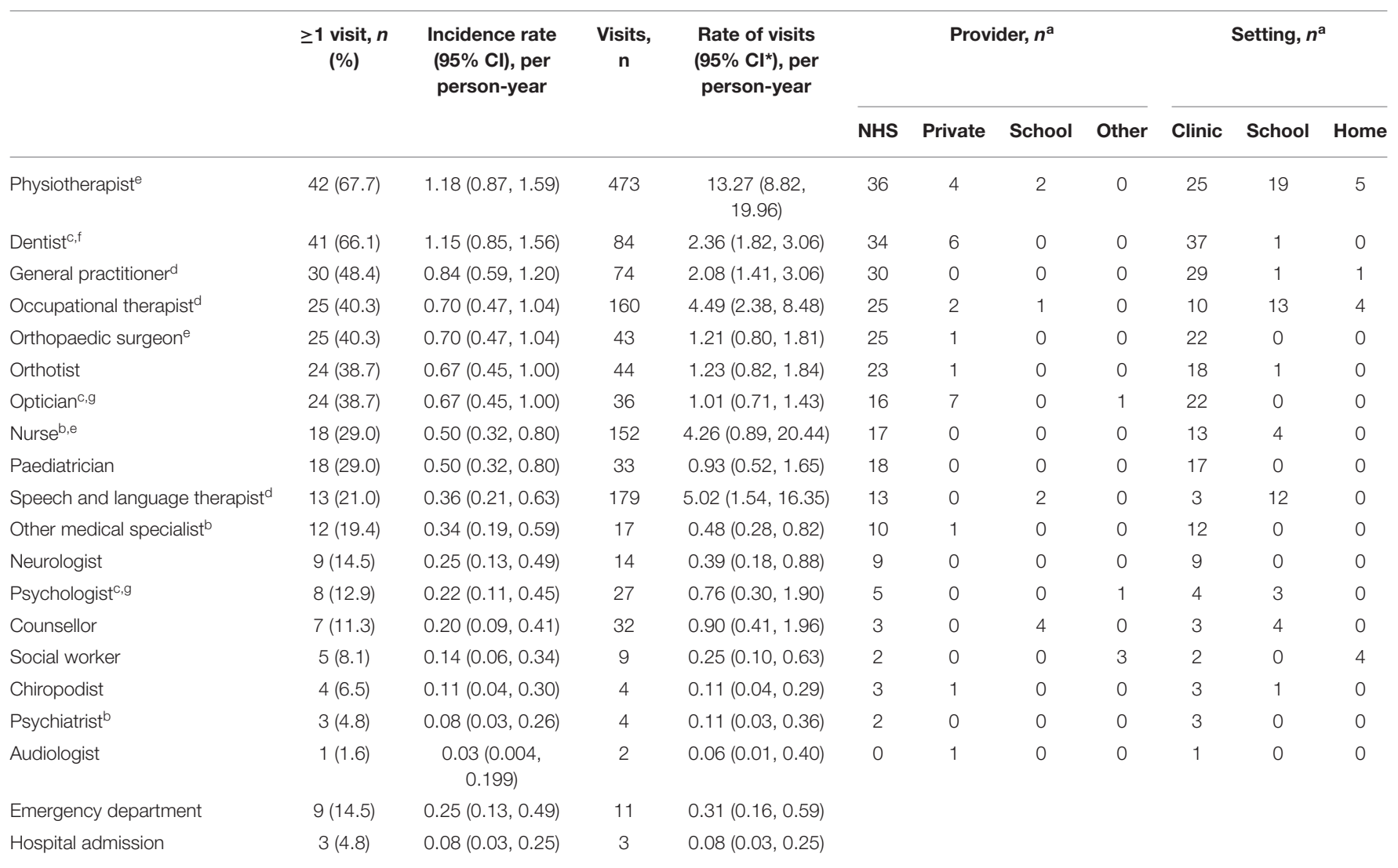

${ }^{*}$ Calculated using robust sandwich estimator of variance; ${ }^{a}$ person may select more than one category; ${ }^{b}$ data on provider missing for one person; ${ }^{c}$ data on provider missing for two people; ${ }^{d}$ data on setting missing for one person; ${ }^{e}$ data on setting missing for three people; ${ }^{f}$ data on setting missing for four people; ${ }^{g}$ data on setting missing for two people.

attended a clinic for most services (Table 2). However, many young people received rehabilitation services in school. Thirteen of 25 participants received occupational therapy in school, 12 of 13 participants received speech and language therapy in school, four of seven participants received counselling in school, and 19 of 42 participants received physiotherapy in school.

\section{Participant Characteristics Associated With at Least One Visit to a Professional Unadjusted Analyses}

Unadjusted associations between participant characteristics and having at least one visit to each professional are reported in Tables 3, 4. In unadjusted analyses, there was some evidence that GMFCS level was positively associated with at least one visit to an occupational therapist $(p=0.030)$, speech and language therapist $(p=0.022)$, nurse $(p=0.078)$ and paediatrician $(p$ $=0.033$ ). People with bilateral $\mathrm{CP}$ were more likely to visit a paediatrician than people with unilateral CP (HR: 3.43, 95\% CI 1.13, 10.43; $p=0.030$ ). People with ASD/ADHD/behaviour impairment were more likely to visit the emergency department (HR: $3.84,95 \%$ CI 1.03, 14.31; $p=0.045$ ). People with epilepsy were more likely to visit an occupational therapist (HR: 2.60, $95 \%$ CI 1.04, 6.50; $p=0.042$ ) and speech and language therapist
(HR 3.70, 95\% CI 1.14, 12.00; $p=0.030$ ). People with learning difficulties were more likely to visit an occupational therapist (HR: $2.79,95 \%$ CI 1.27, 6.15; $p=0.011$ ), speech and language therapist (HR: $3.57,95 \%$ CI 1.17, 10.90; $p=0.026$ ), psychologist or psychiatrist (HR: $4.49,95 \%$ CI 1.12, 17.97; $p=0.034$ ) and optician (HR: $2.26,95 \%$ CI 1.02, 5.03; $p=0.046$ ). People with a speech impairment were more likely to visit a speech and language therapist (HR: 7.70, 95\% CI 2.37, 25.04; $p=0.001$ ). People attending a SEN school were more likely to visit a speech and language therapist than those attending a mainstream school (HR: 4.19, 95\% CI 1.41, 12.46; $p=0.010$ ).

\section{Adjusted Analyses}

As presented in Table 5, in adjusted analyses, people in GMFCS level III remained more likely to visit an occupational therapist (adjusted HR: $4.23,95 \%$ CI 1.25, 14.30; $p=0.020$ ) and nurse (adjusted HR: 4.32, 95\% CI 1.17, 16.00; $p=0.028$ ) than those in level I. There was also weak evidence that people with learning difficulties were more likely to visit an occupational therapist (adjusted HR: 2.35, 95\% CI 1.00, 5.48; $p=0.049$ ) and people with a speech impairment were more likely to visit a speech and language therapist (adjusted HR: 4.14, 95\% CI 0.97, 17.62; $p=0.055$ ). 


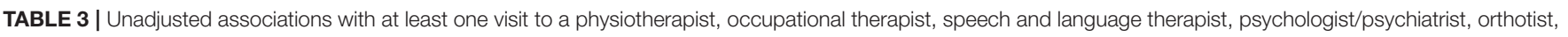
counsellor, optician.

\begin{tabular}{|c|c|c|c|c|c|c|c|}
\hline & $\begin{array}{l}\text { Physiotherapist } \\
\text { unadjusted HR } \\
(95 \% \mathrm{Cl})\end{array}$ & $\begin{array}{c}\text { Occupational } \\
\text { therapist } \\
\text { unadjusted HR } \\
(95 \% \mathrm{Cl})\end{array}$ & $\begin{array}{c}\text { SALT } \\
\text { unadjusted HR } \\
(95 \% \mathrm{Cl})\end{array}$ & $\begin{array}{l}\text { Psychologist/ } \\
\text { Psychiatrist } \\
\text { unadjusted HR } \\
(95 \% \mathrm{Cl})\end{array}$ & $\begin{array}{c}\text { Orthotist } \\
\text { unadjusted HR } \\
(95 \% \mathrm{Cl})\end{array}$ & $\begin{array}{c}\text { Counsellor } \\
\text { unadjusted HR } \\
(95 \% \mathrm{CI})\end{array}$ & $\begin{array}{c}\text { Optician } \\
\text { unadjusted HR } \\
(95 \% \mathrm{Cl})\end{array}$ \\
\hline Age & $0.95(0.84,1.08)$ & $1.02(0.87,1.20)$ & $0.98(0.78,1.22)$ & $0.90(0.68,1.19)$ & $0.91(0.76,1.08)$ & $1.02(0.76,1.37)$ & $1.02(0.87,1.19)$ \\
\hline \multicolumn{8}{|l|}{ Sex } \\
\hline Female & $0.83(0.45,1.54)$ & $1.31(0.60,2.88)$ & $1.40(0.47,4.18)$ & $0.98(0.26,3.63)$ & $0.87(0.38,1.95)$ & $2.98(0.58,15.36)$ & $1.20(0.54,2.67)$ \\
\hline \multicolumn{8}{|l|}{ GMFCS } \\
\hline Level II & $1.39(0.71,2.73)$ & $2.66(1.01,7.00)$ & $4.40(0.91,21.20)$ & $1.26(0.25,6.24)$ & $1.49(0.64,3.45)$ & $2.49(0.46,13.59)$ & $1.03(0.43,2.49)$ \\
\hline Level III & $2.04(0.87,4.78)$ & $4.06(1.31,12.61)$ & $8.50(1.55,46.50)$ & $4.26(0.86,21.15)$ & $0.83(0.18,3.81)$ & $2.16(0.20,23.88)$ & $1.61(0.51,5.07)$ \\
\hline \multicolumn{8}{|l|}{ Distribution } \\
\hline Bilateral & $1.18(0.64,2.17)$ & $0.90(0.41,1.97)$ & $2.20(0.68,7.16)$ & $3.45(0.72,16.59)$ & $1.16(0.52,2.58)$ & $0.39(0.08,2.03)$ & $1.39(0.62,3.13)$ \\
\hline Behaviour impairment & $0.67(0.26,1.71)$ & $0.94(0.32,2.73)$ & $1.45(0.40,5.29)$ & $2.46(0.61,9.83)$ & $0.70(0.21,2.35)$ & $1.92(0.37,9.90)$ & $0.96(0.33,2.81)$ \\
\hline Epilepsy & $1.37(0.58,3.25)$ & $2.60(1.04,6.50)$ & $3.70(1.14,12.00)$ & $1.03(0.13,8.24)$ & $0.36(0.05,2.65)$ & $3.28(0.64,16.90)$ & $1.17(0.35,3.92)$ \\
\hline Learning difficulties & $1.50(0.81,2.79)$ & $2.79(1.27,6.15)$ & $3.57(1.17,10.90)$ & $4.49(1.12,17.97)$ & $1.33(0.58,3.04)$ & $0.89(0.17,4.59)$ & $2.26(1.02,5.03)$ \\
\hline Speech impairment & $1.72(0.91,3.27)$ & $1.91(0.84,4.33)$ & $7.70(2.37,25.04)$ & $1.74(0.44,6.97)$ & $1.15(0.46,2.90)$ & $0.58(0.07,4.83)$ & $1.19(0.47,2.99)$ \\
\hline \multicolumn{8}{|l|}{ School } \\
\hline SEN & $1.30(0.65,2.59)$ & $1.71(0.74,3.96)$ & $4.19(1.41,12.46)$ & $2.92(0.78,10.88)$ & $0.52(0.15,1.74)$ & $2.70(0.60,12.06)$ & $1.21(0.48,3.04)$ \\
\hline
\end{tabular}

Bold text indicates $p<0.010$.

Cl, confidence interval; GMFCS, Gross motor function classification system; SALT, speech and language therapist; SEN, Special education needs.

\section{Participant Characteristics Associated With Number of Visits to a Professional Unadjusted Analyses}

Unadjusted associations between participant characteristics and number of visits to each professional are reported in Tables 6, 7 . Age was negatively associated with visits to a GP (IRR: $0.84,95 \%$ CI $0.72,0.97 ; p=0.019$ ) and positively associated with visits to a nurse (IRR: $1.58,95 \%$ CI 1.13, 2.22; $p=0.008$ ). Compared to people in GMFCS level I, people in GMFCS level II had more visits to a physiotherapist (IRR: $2.80,95 \%$ CI 1.17, 6.68; $p=$ 0.020), occupational therapist (IRR: $4.99,95 \%$ CI 1.48, 16.80; $p=$ 0.009 ), speech and language therapist (IRR: 31.52 (95\% CI 4.69, 211.97; $p<0.001$ ), nurse (IRR: 24.64, 95\% CI 5.11, 118.80; $p$ $<0.001$ ), dentist (IRR: $0.45,95 \%$ CI $0.26,0.79 ; p=0.005$ ) and paediatrician (IRR: 3.71, 95\% CI 1.12, 12.26; $p=0.032$ ). People in GMFCS level III also had more visits to a paediatrician than people in level I (IRR: 7.70, 95\% CI 1.94, 30.50; $p=0.004$ ). People with bilateral CP had more visits to a physiotherapist (IRR: 3.15, 95\% CI 1.42, 6.99; $p=0.005$ ), an occupational therapist (IRR $3.82,95 \%$ CI $1.22,11.92 ; p=0.021$ ), and a paediatrician (IRR: $3.95,95 \%$ CI 1.32, $11.83 ; p=0.014$ ) and less visits to a nurse (IRR: $0.17,95 \%$ CI $0.04,0.78 ; p=0.022$ ) than those with unilateral CP. People with epilepsy had more visits to a speech and language therapist (IRR: 72.61, 95\% CI 16.69, 315.89; $p<0.001$ ), nurse (IRR: 36.97, 95\% CI 7.19, 189.99; $p<0.001$ ) and neurologist (IRR: 5.58, 95\% CI 1.12, 27.71; $p=0.035$ ). People with learning difficulties had more visits to an occupational therapist (IRR: $4.25,95 \%$ CI $1.32,13.62 ; p=0.015)$, psychologist or psychiatrist (IRR: 7.60, 95\% CI 1.38, 41.99; $p=0.020$ ), and nurse (IRR: 14.71, $95 \%$ CI 3.43, 61.13, $p<0.001$ ). People with a speech impairment had more visits to a physiotherapist (IRR: $3.48,95 \%$ CI 1.38, 8.78; $p=0.008$ ), speech and language therapist (IRR: $72.61,95 \% \mathrm{CI}$
16.69, 315.89; $p<0.001$ ), GP (IRR: $2.24,95 \%$ CI 1.02, 4.89; $p=$ 0.044), and nurse (IRR: 21.01, 95\% CI 4.93, 89.60; $p<0.001$ ). People attending a special educational needs school had more visits to a speech and language therapist (IRR: 19.03, 95\% CI 2.40, 145.16; $p=0.004$ ) and nurse (IRR: $19.52,95 \%$ CI 4.16, 91.54; $p<$ $0.001)$ than those in mainstream education.

\section{Adjusted Analyses}

Results from adjusted analyses are reported in Table 8. In adjusted analyses, people with learning difficulties had more visits to an occupational therapist (adjusted IRR: 5.6, 95\% CI 1.7, 18.0; $p=0.004$ ). People with a speech impairment (adjusted IRR: 19.5, 95\% CI 3.9, 97.7; <0.001) and epilepsy (IRR: 6.3, 95\% CI 1.2, 34.4; $p=0.032$ ) had more visits to a speech and language therapist. Age remained negatively associated with rate of GP visits (adjusted IRR: 0.85, 95\% CI 0.74, 0.99; $p=0.035$ ). People with epilepsy had more visits to a nurse (adjusted IRR: 18.1, 95\% CI 2.0, 167.0; $p=0.010$ ) and people in GMFCS level II had more visits to a nurse than people in GMFCS level I (adjusted IRR: 5.4, 95\% CI 1.1, 26.6; $p=0.040$ ).

\section{DISCUSSION}

We aimed to describe patterns of visits to rehabilitation professionals, medical professionals, emergency department visits and hospital admissions among ambulatory young people with CP living in England, and identify factors associated with service use. In our sample, physiotherapists were the most commonly visited professional, with $68 \%$ of young people visiting a physiotherapist at least once, and also the most frequently visited professional, with a visit rate of 13.3 per person-year. A similar proportion of young people visited a dentist at least once. 
TABLE 4 | Unadjusted associations with at least one visit to a general practitioner, nurse, dentist, paediatrician, orthopaedic surgeon, neurologist, other medical specialist and emergency department.

\begin{tabular}{|c|c|c|c|c|c|c|c|c|}
\hline & $\begin{array}{c}\text { GP } \\
\text { unadjusted HR } \\
(95 \% \mathrm{Cl})\end{array}$ & $\begin{array}{c}\text { Nurse } \\
\text { unadjusted HR } \\
(95 \% \mathrm{Cl})\end{array}$ & $\begin{array}{c}\text { Dentist } \\
\text { unadjusted HR } \\
(95 \% \mathrm{Cl})\end{array}$ & $\begin{array}{c}\text { Paediatrician } \\
\text { unadjusted HR } \\
(95 \% \mathrm{Cl})\end{array}$ & $\begin{array}{c}\text { Orthopaedic } \\
\text { surgeon } \\
\text { unadjusted HR } \\
(95 \% \mathrm{Cl})\end{array}$ & $\begin{array}{c}\text { Neurologist } \\
\text { unadjusted HR } \\
(95 \% \mathrm{Cl})\end{array}$ & $\begin{array}{l}\text { Other medical } \\
\text { specialist } \\
\text { unadjusted HR } \\
(95 \% \mathrm{Cl})\end{array}$ & $\begin{array}{c}\text { ED } \\
\text { unadjusted HR } \\
(95 \% \mathrm{Cl})\end{array}$ \\
\hline Age & $0.98(0.85,1.14)$ & $1.04(0.86,1.24)$ & $0.97(0.86,1.10)$ & $0.93(0.76,1.13)$ & $0.92(0.78,1.08)$ & $0.88(0.66,1.17)$ & $0.90(0.70,1.15)$ & $0.88(0.66,1.17)$ \\
\hline \multicolumn{9}{|l|}{ Sex } \\
\hline Female & $1.05(0.51,2.15)$ & $0.96(0.38,2.42)$ & $0.86(0.46,1.60)$ & $1.51(0.59,3.81)$ & $0.69(0.30,1.56)$ & $2.42(0.61,9.69)$ & $0.40(0.11,1.47)$ & $0.96(0.26,3.57)$ \\
\hline \multicolumn{9}{|l|}{ GMFCS } \\
\hline Level II & $0.91(0.40,2.04)$ & $2.00(0.65,6.12)$ & $0.76(0.39,1.47)$ & $2.84(0.87,9.23)$ & $1.24(0.52,2.98)$ & $0.76(0.18,3.16)$ & $1.53(0.47,5.02)$ & $0.43(0.09,2.11)$ \\
\hline Level III & $1.90(0.73,4.96)$ & $4.31(1.25,14.92)$ & $0.73(0.25,2.11)$ & $5.34(1.43,19.90)$ & $2.06(0.70,6.05)$ & $0.88(0.10,7.52)$ & $0.90(0.10,7.67)$ & $0.74(0.09,6.15)$ \\
\hline \multicolumn{9}{|l|}{ Distribution } \\
\hline Bilateral & $1.49(0.72,3.09)$ & $1.55(0.60,3.99)$ & $0.77(0.41,1.42)$ & $3.43(1.13,10.43)$ & $1.25(0.57,2.75)$ & $3.48(0.72,16.73)$ & $1.99(0.60,6.60)$ & $1.24(0.33,4.61)$ \\
\hline Behaviour impairment & $0.74(0.26,2.12)$ & $9.96(0.28,3.33)$ & $1.08(0.45,2.27)$ & $0.97(0.28,3.35)$ & $0.94(0.32,2.75)$ & $1.39(0.29,6.69)$ & $0.96(0.21,4.37)$ & $3.84(1.03,14.31)$ \\
\hline Epilepsy & $1.27(0.44,3.63)$ & $2.35(0.77,7.15)$ & $1.14(0.45,2.90)$ & * & $1.12(0.33,3.74)$ & $2.33(0.48,11.22)$ & * & $1.04(0.13,8.28)$ \\
\hline Learning difficulties & $1.31(0.62,2.76)$ & $2.23(0.88,4.62)$ & $1.28(0.68,2.43)$ & $1.79(0.71,4.53)$ & $1.25(0.55,2.83)$ & $1.81(0.49,6.75)$ & $1.62(0.51,5.11)$ & $1.81(0.49,6.75)$ \\
\hline Speech impairment & $1.77(0.83,3.78)$ & $2.21(0.86,5.70)$ & $0.98(0.47,2.05)$ & $1.71(0.64,4.56)$ & $1.35(0.56,3.23)$ & $1.79(0.45,7.16)$ & $1.76(0.53,5.86)$ & $1.00(0.21,4.82)$ \\
\hline \multicolumn{9}{|l|}{ School } \\
\hline SEN & $1.10(0.47,2.56)$ & $1.38(0.49,3.88)$ & $0.75(0.33,1.69)$ & $1.38(0.49,3.87)$ & $0.92(0.34,2.45)$ & $1.04(0.22,5.03)$ & $1.20(0.32,4.43)$ & $0.45(0.06,3.59)$ \\
\hline
\end{tabular}

Bold text indicates $p<0.010$

*Unable to calculate.

Cl, confidence interval; ED, Emergency department; GMFCS, Gross motor function classification system; GP, General practitioner; SEN, Special education needs 
TABLE 5 | Adjusted associations with at least one visit to an occupational therapist, speech and language therapist, nurse, optician, paediatrician.

\begin{tabular}{|c|c|c|c|c|}
\hline & $\begin{array}{c}\text { Occupational therapy } \\
\text { adjusted HR }(95 \% \mathrm{Cl}) ; \\
\quad p \text {-value }\end{array}$ & $\begin{array}{c}\text { SALT } \\
\text { adjusted HR } \\
\text { (95\% Cl); } \\
p \text {-value }\end{array}$ & $\begin{array}{c}\text { Nurse } \\
\text { adjusted HR } \\
\text { (95\% Cl); } \\
p \text {-value }\end{array}$ & $\begin{array}{l}\text { Paediatrician } \\
\text { adjusted HR } \\
\text { (95\% Cl); } \\
p \text {-value }\end{array}$ \\
\hline \multicolumn{5}{|l|}{ GMFCS } \\
\hline Level II & $2.18(0.80,5.92) ; 0.126$ & $\begin{array}{c}2.40(0.43,13.31) ; \\
0.315\end{array}$ & $\begin{array}{c}1.93(0.63,5.93) \\
0.253\end{array}$ & $\begin{array}{c}2.14(0.61,7.50) \\
0.234\end{array}$ \\
\hline \multicolumn{5}{|l|}{ Distribution } \\
\hline Bilateral & - & - & - & $\begin{array}{c}2.33(0.66,8.18) ; \\
0.188\end{array}$ \\
\hline Behaviour impairment & - & - & - & - \\
\hline Epilepsy & $2.12(0.73,6.15) ; 0.165$ & $\begin{array}{c}2.09(0.35,12.63) ; \\
0.420\end{array}$ & - & \\
\hline \multicolumn{5}{|l|}{ School } \\
\hline SEN & 0.77 (0.30, 1.99); 0.585 & $\begin{array}{c}0.84(0.15,4.75) \\
0.847\end{array}$ & $\begin{array}{c}0.71(0.21,2.36) \\
0.574\end{array}$ & $\begin{array}{c}1.17(0.39,3.50) ; \\
0.776\end{array}$ \\
\hline
\end{tabular}

Cl, confidence interval; GMFCS, Gross motor function classification system; SALT, speech and language therapist; SEN, Special education needs.

TABLE 6 | Unadjusted associations with number of visits to a physiotherapist, occupational therapist, speech and language therapist, psychologist/psychiatrist, orthotist, counsellor, optician.

\begin{tabular}{|c|c|c|c|c|c|c|c|}
\hline & $\begin{array}{c}\text { Physiotherapist } \\
\text { unadjusted IRR } \\
(95 \% \mathrm{Cl})\end{array}$ & $\begin{array}{l}\text { Occupational } \\
\text { therapist } \\
\text { unadjusted IRR } \\
(95 \% \mathrm{Cl})\end{array}$ & $\begin{array}{c}\text { SALT } \\
\text { unadjusted IRR } \\
(95 \% \mathrm{CI})\end{array}$ & $\begin{array}{c}\text { Psychologist/ } \\
\text { Psychiatrist } \\
\text { unadjusted IRR } \\
(95 \% \mathrm{Cl})\end{array}$ & $\begin{array}{c}\text { Orthotist } \\
\text { unadjusted IRR } \\
(95 \% \mathrm{Cl})\end{array}$ & $\begin{array}{c}\text { Counsellor } \\
\text { unadjusted IRR } \\
(95 \% \mathrm{Cl})\end{array}$ & $\begin{array}{c}\text { Optician } \\
\text { unadjusted IRR } \\
(95 \% \mathrm{Cl})\end{array}$ \\
\hline Age & $0.85(0.71,1.01)$ & $0.89(0.70,1.14)$ & $0.74(0.44,1.26)$ & $0.72(0.44,1.19)$ & $0.87(0.73,1.03)$ & $0.95(0.63,1.45)$ & $1.03(0.90,1.18)$ \\
\hline \multicolumn{8}{|l|}{ Sex } \\
\hline Female & $1.11(0.47,2.59)$ & $2.64(0.73,9.51)$ & $3.44(0.47,24.99)$ & $2.14(0.34,13.56)$ & $1.08(0.47,2.49)$ & $2.39(0.26,21.89)$ & $1.02(0.50,2.05)$ \\
\hline Level II & $2.80(1.17,6.68)$ & $4.99(1.48,16.80)$ & $31.5(4.7,212.0)$ & $0.78(0.11,5.75)$ & $1.87(0.81,4.29)$ & $1.47(0.14,15.08)$ & $0.93(0.44,1.99)$ \\
\hline Level III & $3.22(0.97,10.66)$ & $4.33(0.82,22.75)$ & $5.4(0.4,72.8)$ & $0.61(0.03,10.72)$ & $0.43(0.08,2.25)$ & $0.24(0.01,9.74)$ & $0.99(0.34,2.88)$ \\
\hline \multicolumn{8}{|l|}{ Distribution } \\
\hline Bilateral & $3.15(1.42,6.99)$ & $3.82(1.22,11.92)$ & $0.47(0.06,3.45)$ & $1.10(0.17,7.04)$ & $0.96(0.42,2.21)$ & $0.25(0.03,2.18)$ & $1.17(0.58,2.35)$ \\
\hline Behaviour impairment & $0.72(0.22,2.38)$ & $2.57(0.50,13.11)$ & $1.8(0.1,31.3)$ & $6.22(0.68,56.61)$ & $0.67(0.20,2.29)$ & $4.12(0.22,76.47)$ & $1.28(0.53,3.12)$ \\
\hline Epilepsy & $1.38(0.37,5.19)$ & $5.28(0.96,29.04)$ & $26.5(2.3,306.6)$ & $3.94(0.28,55.60)$ & $0.98(0.27,3.61)$ & $4.34(0.17,111.7)$ & $1.03(0.34,3.15)$ \\
\hline SEN & $1.72(0.61,4.90)$ & $3.08(0.74,12.83)$ & $19.0(2.5,145.2)$ & $1.39(0.14,13.99)$ & $0.82(0.29,2.36)$ & $2.92(0.20,42.74)$ & $1.27(0.56,2.85)$ \\
\hline
\end{tabular}

Bold text indicates $p<0.010$.

Cl, confidence interval; GMFCS, Gross motor function classification system; SALT, speech and language therapist; SEN, Special education needs.

All other professions were visited by less than half of participants during the study period. Despite only $21 \%$ of young people visiting a speech and language therapist and only $40 \%$ visiting on occupational therapist, these were the second and third most frequently visited professionals, with visit rates of 5.0 and 4.5 per person-year, respectively. In adjusted analyses, GMFCS level, age and presence of epilepsy, learning difficulties and speech impairment were associated with having at least one visit and number of visits to a range of professionals.

Few studies that report service use among young people with $\mathrm{CP}$ have examined the range of services examined in this study. Estimates for the proportion of young people visiting an occupational therapist (44\%) and speech and language therapist (19\%) in the Netherlands (4) were almost identical to estimates 
TABLE 7 | Unadjusted associations with number of visits to a general practitioner, nurse, dentist, paediatrician, orthopaedic surgeon, neurologist, other medical specialist and emergency department.

\begin{tabular}{|c|c|c|c|c|c|c|c|c|}
\hline & $\begin{array}{c}\text { GP } \\
\text { unadjusted IRR } \\
(95 \% \mathrm{Cl})\end{array}$ & $\begin{array}{c}\text { Nurse } \\
\text { unadjusted IRR } \\
(95 \% \mathrm{Cl})\end{array}$ & $\begin{array}{c}\text { Dentist } \\
\text { unadjusted IRR } \\
(95 \% \mathrm{Cl})\end{array}$ & $\begin{array}{c}\text { Paediatrician } \\
\text { unadjusted IRR } \\
(95 \% \mathrm{Cl})\end{array}$ & $\begin{array}{l}\text { Orthopaedic } \\
\text { surgeon } \\
\text { unadjusted IRR } \\
(95 \% \mathrm{Cl})\end{array}$ & $\begin{array}{c}\text { Neurologist } \\
\text { unadjusted IRR } \\
(95 \% \mathrm{Cl})\end{array}$ & $\begin{array}{l}\text { Other medical } \\
\text { specialist } \\
\text { unadjusted IRR } \\
(95 \% \mathrm{Cl})\end{array}$ & $\begin{array}{c}\text { ED } \\
\text { unadjusted IRR } \\
(95 \% \mathrm{Cl})\end{array}$ \\
\hline Age & $0.84(0.72,0.97)$ & $1.58(1.13,2.22)$ & $0.98(0.88,1.09)$ & $0.92(0.74,1.14)$ & $0.89(0.74,1.07)$ & $0.80(0.54,1.19)$ & $0.87(0.68,1.11)$ & $0.88(0.66,1.18)$ \\
\hline \multicolumn{9}{|l|}{ Sex } \\
\hline Female & $1.35(0.64,2.81)$ & $0.11(0.02,0.53)$ & $0.71(0.42,1.20)$ & $2.30(0.82,6.41)$ & $1.01(0.44,2.31)$ & $2.98(0.67,13.18)$ & $0.53(0.16,1.75)$ & $1.08(0.29,3.95)$ \\
\hline \multicolumn{9}{|l|}{ GMFCS } \\
\hline Level II & $1.24(0.56,2.73)$ & $24.6(5.1,118.8)$ & $0.45(0.26,0.79)$ & $3.71(1.12,12.26)$ & $1.16(0.47,2.82)$ & $0.48(0.10,2.36)$ & $0.97(0.29,3.26)$ & $0.29(0.06,1.42)$ \\
\hline Level III & $1.12(0.36,3.44)$ & $3.4(0.4,30.7)$ & $0.64(0.30,1.36)$ & $7.70(1.94,30.50)$ & $1.34(0.40,4.50)$ & $0.35(0.02,4.54)$ & $0.81(0.13,4.95)$ & $0.43(0.05,3.72)$ \\
\hline \multicolumn{9}{|l|}{ Distribution } \\
\hline Bilateral & $1.85(0.89,3.85)$ & $0.17(0.04,0.78)$ & $0.91(0.54,1.53)$ & $3.95(1.32,11.83)$ & $1.24(0.54,2.83)$ & $1.32(0.30,5.87)$ & $1.30(0.42,4.06)$ & $0.79(0.22,2.88)$ \\
\hline Behaviour impairment & $1.15(0.42,3.17)$ & $0.17(0.02,1.90)$ & $1.51(0.79,2.87)$ & $0.53(0.11,2.64)$ & $1.72(0.62,4.74)$ & $3.87(0.77,19.53)$ & $1.14(0.25,5.18)$ & $3.03(0.80,11.45)$ \\
\hline Epilepsy & $2.62(0.99,6.95)$ & $37.0(7.2,190.0)$ & $0.62(0.24,1.61)$ & * & $1.43(0.43,4.67)$ & $5.58(1.12,27.71)$ & * & $0.80(0.09,7.37)$ \\
\hline Learning difficulties & $1.72(0.82,3.64)$ & $14.7(3.5,61.1)$ & $0.97(0.55,1.69)$ & $1.88(0.66,5.36)$ & $1.41(0.61,3.26)$ & $2.56(0.60,11.00)$ & $1.42(0.45,4.50)$ & $1.18(0.31,4.51)$ \\
\hline Speech impairment & $2.24(1.02,4.89)$ & $21.0(4.9,89.6)$ & $0.76(0.40,1.45)$ & $2.15(0.70,6.61)$ & $0.62(0.22,1.75)$ & $0.86(0.14,5.10)$ & $1.34(0.38,4.74)$ & $0.74(0.14,3.87)$ \\
\hline \multicolumn{9}{|l|}{ School } \\
\hline SEN & $1.77(0.75,4.17)$ & $19.5(4.2,91.5)$ & $0.68(0.34,1.36)$ & $2.15(0.67,6.91)$ & $0.60(0.20,1.81)$ & $0.63(0.09,4.55)$ & $1.57(0.44,5.66)$ & $0.38(0.05,3.25)$ \\
\hline
\end{tabular}

Bold text indicates $p<0.010$

*Unable to calculate.

Cl, confidence interval; ED, Emergency department; GMFCS, Gross motor function classification system; GP, General practitioner; SEN, Special education needs 
TABLE 8 | Adjusted associations with number of visits to a physiotherapist, occupational therapist, speech and language therapist, general practitioner, nurse, paediatrician.

\begin{tabular}{|c|c|c|c|c|c|c|}
\hline & $\begin{array}{c}\text { Physiotherapist } \\
\text { adjusted IRR ( } 95 \% \\
\text { Cl); } p \text {-value }\end{array}$ & $\begin{array}{c}\text { Occupational } \\
\text { therapist } \\
\text { adjusted IRR ( } 95 \% \\
\text { CI); } p \text {-value }\end{array}$ & $\begin{array}{c}\text { SALT } \\
\text { adjusted IRR ( } 95 \% \\
\text { CI); } p \text {-value }\end{array}$ & $\begin{array}{c}\text { GP } \\
\text { adjusted IRR (95\% } \\
\text { Cl); } p \text {-value }\end{array}$ & $\begin{array}{c}\text { Nurse } \\
\text { adjusted IRR (95\% } \\
\text { CI); } p \text {-value }\end{array}$ & $\begin{array}{c}\text { Paediatrician } \\
\text { adjusted IRR (95\% } \\
\text { Cl); } p \text {-value }\end{array}$ \\
\hline Age & $\begin{array}{c}0.82(0.66,1.01) \\
0.067\end{array}$ & - & - & $\begin{array}{c}0.85(0.74,0.99) \\
0.035\end{array}$ & $\begin{array}{c}1.05(0.77,1.42) \\
0.760\end{array}$ & \\
\hline \multicolumn{7}{|l|}{ Sex } \\
\hline Female & - & - & - & - & $\begin{array}{c}0.24(0.06,0.96) \\
0.043\end{array}$ & \\
\hline \multicolumn{7}{|l|}{ GMFCS } \\
\hline Level II & $\begin{array}{c}1.57(0.61,4.05) \\
0.353\end{array}$ & $\begin{array}{c}3.3(0.8,14.7) \\
0.113\end{array}$ & $\begin{array}{c}1.9(0.3,11.3) \\
0.481\end{array}$ & - & $\begin{array}{c}5.4(1.1,26.6) \\
0.040\end{array}$ & $\begin{array}{c}2.92(0.86,9.90) \\
0.086\end{array}$ \\
\hline Level III & $\begin{array}{c}2.23(0.56,8.85) \\
0.254\end{array}$ & $\begin{array}{c}7.2(0.9,56.9) \\
0.060\end{array}$ & $\begin{array}{c}0.9(0.1,10.3) \\
0.926\end{array}$ & - & $\begin{array}{c}8.8(0.5,142.6) \\
0.124\end{array}$ & $\begin{array}{c}3.9(0.8,19.5) \\
0.104\end{array}$ \\
\hline \multicolumn{7}{|l|}{ Distribution } \\
\hline Bilateral & $\begin{array}{c}1.64(0.65,4.15) \\
0.298\end{array}$ & $\begin{array}{c}0.81(0.17,3.78) \\
0.787\end{array}$ & - & $\begin{array}{c}1.69(0.84,3.40) \\
0.145\end{array}$ & $\begin{array}{c}0.95(0.16,5.54) \\
0.950\end{array}$ & $\begin{array}{c}2.45(0.75,8.04) \\
0.140\end{array}$ \\
\hline Behaviour impairment & - & - & - & - & - & \\
\hline Epilepsy & - & $\begin{array}{c}3.8(0.6,22.3) \\
0.142\end{array}$ & $\begin{array}{c}6.3(1.2,34.4) ; \\
0.032\end{array}$ & $\begin{array}{c}2.08(0.76,5.66) \\
0.153\end{array}$ & $\begin{array}{c}18.1(2.0,167.0) \\
0.010\end{array}$ & \\
\hline Learning difficulties & - & $\begin{array}{c}5.6(1.7,18.0) \\
0.004\end{array}$ & - & - & $\begin{array}{c}1.53(0.35,6.63) \\
0.568\end{array}$ & \\
\hline Speech impairment & $\begin{array}{c}2.38(0.87,6.46) \\
0.090\end{array}$ & $\begin{array}{c}2.43(0.60,9.91) \\
0.215\end{array}$ & $\begin{array}{c}19.5(3.9,97.7) \\
<0.001\end{array}$ & $\begin{array}{c}1.38(0.58,3.28) \\
0.465\end{array}$ & $\begin{array}{c}1.8(0.3,10.7) \\
0.493\end{array}$ & \\
\hline \multicolumn{7}{|l|}{ School } \\
\hline SEN & $\begin{array}{c}1.31(0.41,4.26) ; \\
0.649\end{array}$ & $\begin{array}{c}0.75(0.17,3.24) \\
0.697\end{array}$ & $\begin{array}{c}3.0(0.7,13.8) \\
0.156\end{array}$ & $\begin{array}{c}1.21(0.45,3.30) ; \\
0.703\end{array}$ & $\begin{array}{c}0.44(0.05,3.63) \\
0.443\end{array}$ & $\begin{array}{c}1.45(0.44,4.80) \\
0.546\end{array}$ \\
\hline
\end{tabular}

Cl, confidence interval; GMFCS, Gross motor function classification system; GP, General practitioner; SALT, speech and language therapist; SEN, Special education needs.

from this study. The proportion of young people visiting a physiotherapist was higher than that reported among 13-18 year olds in the United States (59.4\% over 12 months) (13) and $12-$ 19 year olds in the Netherlands (51.8\% over 6 months) (4). In contrast, the proportion visiting a physiotherapist, occupational therapist and speech and language therapist was lower in this study than in a study of young people aged 12-18 years in GMFCS levels IV and V in Northern Ireland (92, 62, and 39\%, respectively) (5).

Less than half of young people visited a medical professional during the study period. The proportion visiting an orthopaedic surgeon, at $40 \%$, was lower than that reported among young people in GMFCS levels IV and V in Northern Ireland (64\% over 6 months) (5). While the difference may be partly explained by difference in GMFCS level, this study also excluded individuals who had received orthopaedic surgery of the lower limbs in the past year, which may have resulted in a biased sample. A third of ambulatory young people in France visited a neurologist (20), which is much higher than the $14.5 \%$ of our sample. The higher proportion of people visiting a neurologist in France may partly be explained by a higher prevalence of epilepsy in the sample. Compared to other professions, the number of young people visiting a psychologist or psychiatrist was small. Only $5 \%$ of young people in this study visited a psychiatrist, which is identical to the proportion of ambulatory young people who visited a psychiatrist in France (20). Thirteen percent visited a psychologist, which is similar to the $15 \%$ who visited a psychologist in the study in the Netherlands (4) but higher than the $3 \%$ reported in the study in Northern Ireland (5).

The relatively small number of people visiting a paediatrician or neurologist suggests that a medical professional is not coordinating their care. Only $15 \%$ of young people with CP in the UK reported having a person they can easily contact to support with co-ordination of care (21). Further, in the UK, nearly half of young people with $\mathrm{CP}$ are discharged from paediatric services to their GP, in the absence of dedicated adult services (11). In this study, the rate of visits to GPs, at approximately 2 per person year, is nearly identical to that reported from a recent analysis of primary care data of children aged 10-14 years in England (12). The same analysis indicated that the rate of visits increased to approximately 2.5 per person year at age 15-19 years and 3.5 per person year at 20-24 years (12), which corresponds with the GP becoming the co-ordinator of care. We however, found that the rate of visits to GPs declined with age. This may be because we included those in GMFCS levels I-III, who potentially do not report health issues until adulthood, and thus there may be a delay between being discharged from paediatric services and requiring access to adult services via their GP. A study in France similarly observed that the proportion of ambulatory young people visiting a GP was lower in those aged 12-17 years 
compared to those aged 6-11 years but increased in the group aged 18 years and older (20). This potential decline in visits to a GP in adolescence may result in the GP being unfamiliar with the young person's medical history and contribute to the lack of continuity of care reported by young adults with CP (6).

We found limited data on service use among young people with other long-term conditions in the UK or internationally. A report from the UK Cystic Fibrosis Registry indicated that $96 \%$ of people with cystic fibrosis received an annual review in 2019 (22). Seventy-eight percent of people with cystic fibrosis under 18 received any form of positive expiratory pressure and $66 \%$ received an exercise intervention from a physiotherapist (22). Among 175 children with attention deficit/hyperactivity disorder (ADHD) in the UK, use of medical services was higher compared to that in our study, with 59\% visiting a hospital, $69 \%$ visiting a doctor, and $47 \%$ visiting a psychologist in the past 6 months (23). Not unlike our findings, a study of 122 young people aged 12-17 years with autism spectrum disorder in Germany found that the dentist/orthodontist was the most commonly visited professional over 12 months (24). However, unlike our study, the paediatrician was the second most commonly visited professional, with $50 \%$ visiting the paediatrician at least once in the past year. Nearly $30 \%$ of young people also reported visiting a ASD outpatient clinic in the past year. The higher proportion of young people visiting specialist services may explain why only $27 \%$ visited the GP in the past year (24), in contrast to $48 \%$ of our sample. Conversely, use of physiotherapy, speech and language therapy, and occupational therapy was higher among young people with CP than ASD (24), which concurs with a study that directly compared therapy use between children with CP and ASD in the United States (25).

In unadjusted analyses, we found that GMFCS level was positively associated with having at least one visit to an occupational therapist and speech and language therapist, and having more visits to a physiotherapist and occupational therapist. This is consistent with previous studies that found severity of motor impairment was positively associated with using physiotherapy, occupational therapy, and speech and language therapy $(4,13)$. However, in adjusted analyses GMFCS level was only associated with visiting an occupational therapist and nurse. In contrast to a previous study (4), we did not find that children in specialist education settings were more likely to receive services. However, this may be because a relatively low proportion of people in this study attended a special educational needs school. In agreement with a study from the Netherlands (4), unadjusted analyses indicated that young people with learning difficulties were more likely to visit a psychologist or psychiatrist. However, in our adjusted analyses, learning difficulties were only associated with visiting an occupational therapist, and not a psychologist or psychiatrist.

While this study shows the proportion of young people with $\mathrm{CP}$ using services is generally low, it does not indicate the needs of young people or unmet need. However, some findings do align with needs reported by a group of young people with $\mathrm{CP}$ from the UK, the majority of whom were in GMFCS levels IIII (11). Twenty-two percent of young people reported needs relating to speech (11), 42\% report needs relating to bone and joint problems, and $45 \%$ report needs relating to eyesight. In this study $21 \%$ of young people used speech and language therapy, $40 \%$ visited an orthopaedic surgeon, and 38\% visited an optician. Although the findings may be used to support future service planning, a significant proportion of ambulatory young people with CP in the UK report unmet health needs, which also need to be considered when allocating resources (11). In particular, between 35 and $60 \%$ of young people and parents report that needs are not being met in relation to bone and joint problems, pain, and speech (11). There is also a trend toward an increase in unmet health needs with age, highlighting that people with $\mathrm{CP}$ continue to require access to services as they transition to adulthood (11).

Understanding existing service use and factors associated with service use is pertinent in order to identify gaps in health care, and to develop services. Despite the relatively high incidence of CP compared to other childhood-onset conditions, there is limited information regarding service use among young people with $\mathrm{CP}$ in the NHS. The breadth of health services accessed by young people with CP demonstrates the complexity of the condition and the need for a co-ordinated approach to developing services. In particular, it highlights the lack of condition-specific specialist services available for young people with CP. Combining this data with currently available and future data on the unmet needs of young people with $\mathrm{CP}$ is warranted to inform service development that meets the needs of people with CP throughout their life.

A limitation of this study is that participants were recruited largely through the physiotherapists in the NHS, hence potentially biasing the sample toward individuals who visit physiotherapists frequently. However, not all participants were recruited through the NHS. Further, participants recruited through the NHS received information about the study even if they were not currently attending physiotherapy. Importantly our study has a cross-sectional design and therefore we cannot make inferences regarding the change in service use among young people with $\mathrm{CP}$ over time. Findings are also limited to young people with spastic CP in GMFCS levels I-III without severe intellectual disability. For context, $\sim 92 \%$ of people have spastic CP and $\sim 75 \%$ are in GMFCS levels I-III in the UK (16). As the data for this study were collected as part of a trial examining the effects of resistance training, young people were excluded if they had orthopaedic surgery of the lower limbs in the past 12 months, botulinum toxin type A injections or serial casting in the past 6 months. Individuals in receipt of these interventions are likely more frequent users of health services than individuals included in our study, and therefore service use in our sample may be an underestimation of use in the population. Finally, we may not have found associations between participant characteristics and service use because the number of people who visited some services was relatively small.

Despite the relatively high prevalence of $\mathrm{CP}$, there is a stark lack of data on health service use. Data on patterns of use may be helpful for planning future services. Generally the proportion of young people with CP using services was low. In the context of limited resources, data on service use in 
combination with data on unmet health needs, may support the reorganisation of services to maximise benefits to young people with CP.

\section{DATA AVAILABILITY STATEMENT}

The datasets presented in this article are not readily available because the datasets generated during and/or analysed during the current study are not expected to be made available publicly, as consent was not obtained to publish the anonymised data. Requests to access the datasets should be directed to Jennifer Ryan, jenniferryan@rcsi.com.

\section{ETHICS STATEMENT}

Approval was obtained from Brunel University London's College of Health and Life Sciences Research Ethics Committee and the Surrey Borders Research Ethics Committee (ref:

\section{REFERENCES}

1. Blair E, Langdon K, McIntyre S, Lawrence D, Watson L. Survival and mortality in cerebral palsy: observations to the sixth decade from a data linkage study of a total population register and National Death Index. BMC Neurol. (2019) 19:111. doi: 10.1186/s12883-019-1343-1

2. Rosenbaum P, Paneth N, Leviton A, Goldstein M, Bax M, Damiano D, et al. A report: the definition and classification of cerebral palsy April 2006. Dev Med Child Neurol Suppl. (2007) 109:8-14.

3. Carter B, Bennett CV, Jones H, Bethel J, Perra O, Wang T, et al. Healthcare use by children and young adults with cerebral palsy. Dev Med Child Neurol. (2021) 63:75-80. doi: 10.1111/dmcn.14536

4. Majnemer A, Shikako-Thomas K, Lach L, Shevell M, Law M, Schmitz $\mathrm{N}$, et al. Rehabilitation service utilization in children and youth with cerebral palsy. Child Care Health Dev. (2014) 40:275-82. doi: 10.1111/cch. 12026

5. McDowell BC, Duffy C, Parkes J. Service use and family-centred care in young people with severe cerebral palsy: a population-based, cross-sectional clinical survey. Disabil Rehabil. (2015) 37:2324-9. doi: 10.3109/09638288.2015.10 19649

6. Bagatell N, Chan D, Rauch KK, Thorpe D. “Thrust into adulthood": transition experiences of young adults with cerebral palsy. Disabil Health J. (2017) 10:80-6. doi: 10.1016/j.dhjo.2016.09.008

7. Ryan JM, Cameron MH, Liverani S, Smith KJ, O'Connell N, Peterson $\mathrm{MD}$, et al. Incidence of falls among adults with cerebral palsy: a cohort study using primary care data. Dev Med Child Neurol. (2020) 62:47782. doi: $10.1111 / \mathrm{dmcn} .14444$

8. Ryan JM, Peterson MD, Matthews A, Ryan N, Smith KJ, O'Connell NE, et al. Noncommunicable disease among adults with cerebral palsy: a matched cohort study. Neurology. (2019) 93:e1385-96. doi: 10.1212/WNL.0000000000 008199

9. Smith KJ, Peterson MD, O'Connell NE, Victor C, Liverani S, Anokye $\mathrm{N}$, et al. Risk of depression and anxiety in adults with cerebral palsy. JAMA Neurol. (2019) 76:294-300. doi: 10.1001/jamaneurol.201 8.4147

10. O'Connell NE, Smith KJ, Peterson MD, Ryan N, Liverani S, Anokye $\mathrm{N}$, et al. Incidence of osteoarthritis, osteoporosis and inflammatory musculoskeletal diseases in adults with cerebral palsy: a populationbased cohort study. Bone. (2019) 125:30-5. doi: 10.1016/j.bone.2019. 05.007

11. Solanke F, Colver A, McConachie H. Are the health needs of young people with cerebral palsy met during transition from child to adult health care? Child Care Health Dev. (2018) 44:355-63. doi: 10.1111/cch.12549
15/LO/0843). Participants 16 years and over provided written consent. Participants under 16 years provided assent, and parents of participants under 16 years provided written consent.

\section{AUTHOR CONTRIBUTIONS}

JR, CK, and GL conceived and designed the analysis. JR, GL, NT, and MN collected the data. JR and GL performed the analysis and drafted the manuscript. NT, CK, and MN provided critical feedback and contributed to the final manuscript. All authors contributed to the article and approved the submitted version.

\section{FUNDING}

Action Medical Research and the Chartered Society of Physiotherapy Charitable Trust have jointly funded this project, and it was supported by a generous grant from The Henry Smith Charity (GN2340).

12. The National Confidential Enquiry into Patient Outcome and Death. Each and Every Need. London: National Confidential Enquiry into Patient Outcome and Death (2018).

13. Liljenquist K, O'Neil ME, Bjornson KF. Utilization of physical therapy services during transition for young people with cerebral palsy: a call for improved care into adulthood. Phys Ther. (2018) 98:796-803. doi: 10.1093/ptj/ pzy068

14. Meehan E, Reid SM, Williams K, Freed GL, Sewell JR, Vidmar S, et al. Hospital admissions in children with cerebral palsy: a data linkage study. Dev Med Child Neurol. (2017) 59:512-9. doi: 10.1111/dmcn. 13350

15. Meehan E, Williams K, Reid SM, Freed GL, Babl FE, Sewell JR, et al. Comparing emergency department presentations among children with cerebral palsy with general childhood presentations: a data linkage study. Dev Med Child Neurol. (2017) 59:1188-95. doi: 10.1111/dmcn. 13518

16. McConnell K, Livingstone E, Perra O, Kerr C. Population-based study on the prevalence and clinical profile of adults with cerebral palsy in Northern Ireland. BMJ Open. (2021) 11:e044614. doi: 10.1136/bmjopen-2020-0 44614

17. Ryan JM, Lavelle G, Theis N, Noorkoiv M, Kilbride C, Korff T, et al. Progressive resistance training for adolescents with cerebral palsy: the STAR randomized controlled trial. Dev Med Child Neurol. (2020) 62:128393. doi: 10.1111/dmcn.14601

18. Palisano R, Rosenbaum P, Walter S, Russell D, Wood E, Galuppi B. Development and reliability of a system to classify gross motor function in children with cerebral palsy. Dev Med Child Neurol. (1997) 39:21423. doi: 10.1111/j.1469-8749.1997.tb07414.x

19. Beecham JKM. Costing Psychiatric Interventions. Measuring Mental Health Needs. 2nd ed. London: Royal College of Psychiatrists (2001). p. 1-22.

20. Roquet M, Garlantezec R, Remy-Neris O, Sacaze E, Gallien P, Ropars J, et al. From childhood to adulthood: health care use in individuals with cerebral palsy. Dev Med Child Neurol. (2018) 60:1271-7. doi: 10.1111/dmcn. 14003

21. Colver A, Pearse R, Watson RM, Fay M, Rapley T, Mann KD, et al. How well do services for young people with long term conditions deliver features proposed to improve transition? BMC Health Serv Res. (2018) 18:337. doi: 10.1186/s12913-01 8-3168-9

22. UK Cystic Fibrosis Registry. Annual Data Report 2019. Available online at https://www.cysticfibrosis.org.uk/sites/default/files/2020-12/2019 \%20Registry\%20Annual\%20Data\%20report_Sep\%202020.pdf (accessed March 29, 2021). 
23. Fibert $\mathrm{P}$, Relton $\mathrm{C}$. What families in the UK use to manage attention deficit/hyperactivity disorder (ADHD): a survey of resource use. BMJ Paediatr Open. (2020) 4:e000771. doi: 10.1136/bmjpo-2020-000771

24. Hofer J HF, Dorks M, Kamp-becker I, Kupper C, Poustka L, Roepke S, et al. Health services use and costs in indivifuals with autism spectrum disorder in Germany: results from a survey in ASD outpatient clinics. J Autism Dev Disord. (2021). doi: 10.1007/s10803-021-04955-4. [Epub ahead of print].

25. Benevides TW, Carretta HJ, Ivey CK, Lane SJ. Therapy access among children with autism spectrum disorder, cerebral palsy, and attention-deficithyperactivity disorder: a population-based study. Dev Med Child Neurol. (2017) 59:1291-8. doi: $10.1111 / \mathrm{dmcn} .13560$
Conflict of Interest: The authors declare that the research was conducted in the absence of any commercial or financial relationships that could be construed as a potential conflict of interest.

Copyright $(02021$ Ryan, Lavelle, Theis, Kilbride and Noorkoiv. This is an open-access article distributed under the terms of the Creative Commons Attribution License (CC $B Y)$. The use, distribution or reproduction in other forums is permitted, provided the original author(s) and the copyright owner(s) are credited and that the original publication in this journal is cited, in accordance with accepted academic practice. No use, distribution or reproduction is permitted which does not comply with these terms. 